\section{Statin alternative awaits go-ahead}

As Esperion Therapeutics awaited the US Food and Drug Administration's decision on its first-in-class drug, slated for 21 February, cardiologists welcomed trial evidence that the drug significantly reduces low-density lipoprotein cholesterol (LDL-C) in patients at high risk of cardiovascular disease. Esperion and investigators reported in JAMA the results from a phase 3 trial in 779 patients with atherosclerotic cardiovascular disease or heterozygous familial hypercholesterolemia. At 12 weeks, the experimental drug bempedoic acid added to maximally tolerated statins lowered LDL-C by $15.1 \%$ from baseline, whereas in the control group of patients on background lipid-lowering therapy LDL-C level increased by $2.4 \%$. In a pooled analysis of four pivotal trials, reported in November at the American Heart Association (AHA) meeting, bempedoic acid lowered LDL-C by $18 \%$ compared with placebo in patients on background statin therapy, and by $25 \%$ compared with placebo in statin-intolerant patients not on statin background therapy. A cardiovascular outcomes trial is ongoing, with results anticipated by 2022 .

Bempedoic acid is an inhibitor of adenosine-citrate lyase, an enzyme in the lipid biosynthesis pathway that acts upstream of HMG-CoA reductase, the target of statins. It is under review with the FDA both as a monotherapy and as a combination tablet with generic lipidlowering agent ezetimibe, for patients with elevated LDL-C. Should it be approved, the oral drug will face a crowded market, dominated by cheap, convenient, generic statins. Esperion's agent will also compete with two approved subcutaneously injected PCSK9-targeted antibodies, which facilitate the clearance of LDL cholesterol from the blood. Sales of these antibodies have disappointed to date, in part due to their high cost. But Novartis acquired The Medicines Company in November over hopes for a PCSK9directed siRNA drug, currently under review at the FDA. An orally available bempedoic acid could shake things up further, especially if priced below PCSK9 modulators. Novartis and Akcea Therapeutics are also recruiting patients into a phase 3 trial of TQJ230, an antisense candidate that cuts lipoprotein(a) levels.

Published online: 7 February 2020

https://doi.org/10.1038/s41587-020-0424-4

\section{Drugmakers turn sights on scarred lungs}

\author{
A 'master regulator' of fibrosis, pentraxin, is in drugmakers' sights, but \\ drivers and mechanisms of the resulting remorseless and fatal lung disease \\ remain unsolved.
}

R oche is spending $\$ 1.4$ billion to snap up a biotech company with a first-in-class drug candidate to treat fibrotic lungs. PRM-151, a recombinant version of the endogenous human protein pentraxin-2 pioneered by Promedior, has shown promising clinical activity in phase 2 studies, giving Roche another shot on goal as it faces looming competition for its idiopathic pulmonary fibrosis (IPF) small-molecule drug Esbriet (pirfenidone). Promedior's agent is part of a diverse and steadily maturing IPF pipeline, which is notable for the wide variety of mechanisms being explored (Table 1). The variety reflects the fact that much remains unknown about IPF and how best to tackle it. For patients, the approvals of Esbriet and of Boehringer Ingelheim's small molecule Ofev (nintedanib) have made a real difference, but new and better agents are still urgently needed. "The reality is our patients are still dying of respiratory failure," says
Toby Maher, professor of interstitial lung disease at Imperial College London and consultant respiratory physician at Royal Brompton Hospital.

IPF is a remorseless disease. It begins with an inflammatory phase, followed by a chronic fibrosis stage during which damaged epithelial cells in the tissue surrounding the alveoli (the lungs' air sacs) undergo apoptosis and are replaced by infiltrating pro-fibrotic fibroblasts, leading to a buildup of scar tissue and a fatal loss of lung function. Its precise triggers are unknown, but environmental toxins and genetic risk factors can predispose individuals to developing the condition. Even though the two approved drugs reduce the annual rate of decline in lung function by $\sim 50 \%$ and lower the frequency of life-threatening acute exacerbations, they neither reverse nor halt the pathological process. Patients have median survival of three to five years

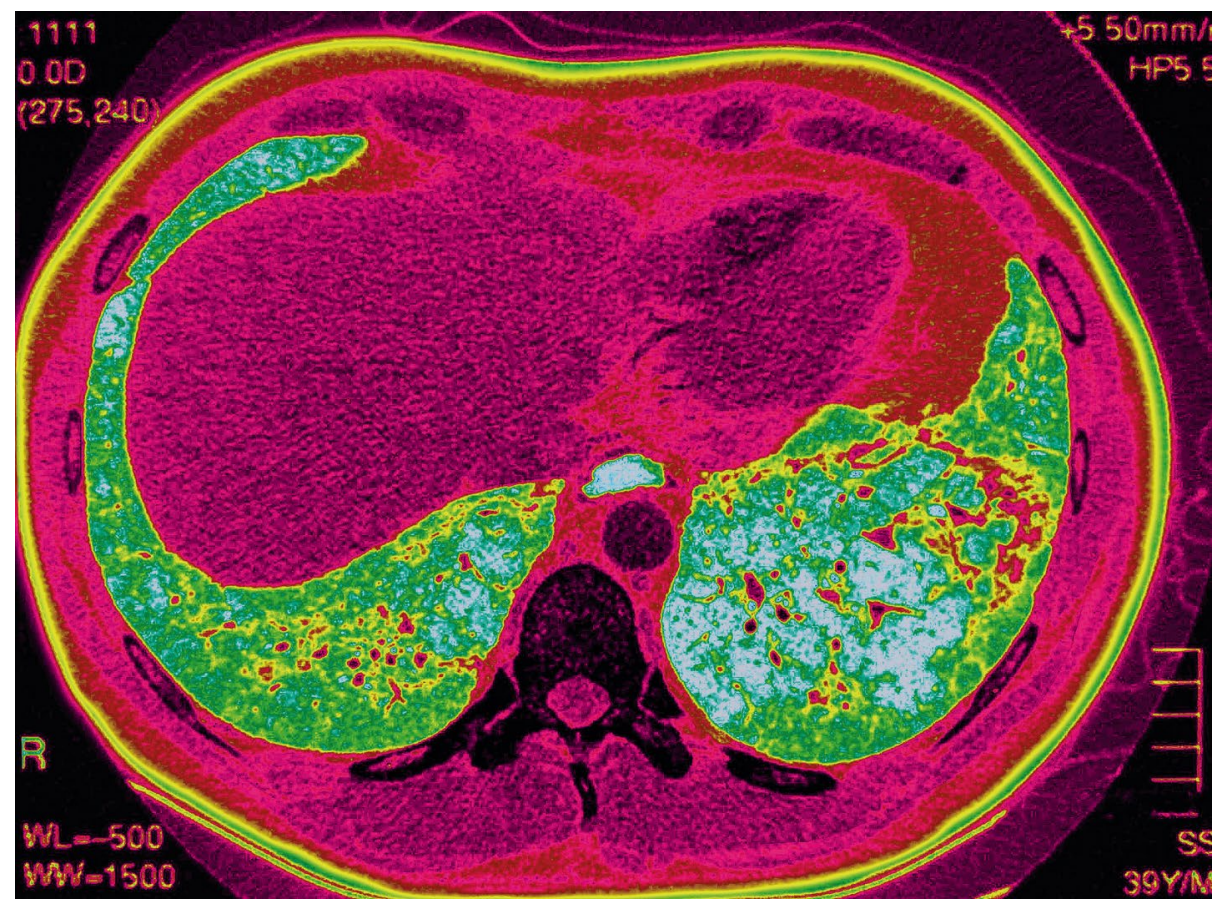

A thoracic scan reveals pulmonary fibrosis, a fatal, age-associated lung disease characterized by irreversible lung scarring. Credit: James Cavallini/Science Source 